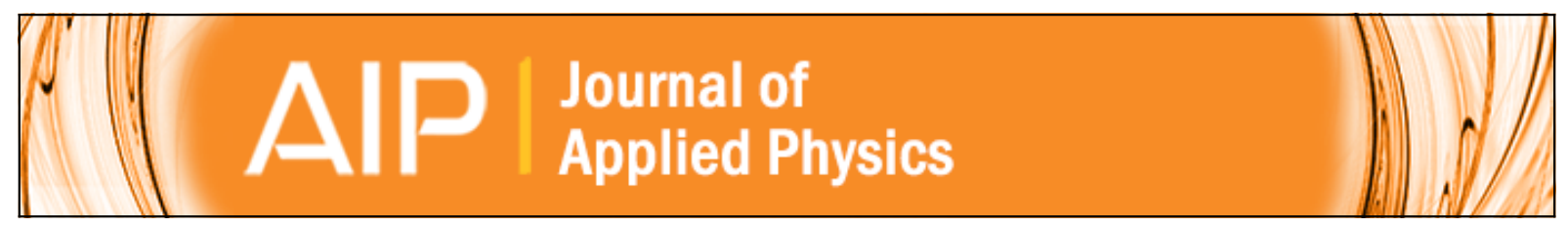

\title{
Electron collision cross sections for tetraethoxysilane
}

W. Lowell Morgan, Carl Winstead, and Vincent McKoy

Citation: Journal of Applied Physics 92, 1663 (2002); doi: 10.1063/1.1491024

View online: http://dx.doi.org/10.1063/1.1491024

View Table of Contents: http://scitation.aip.org/content/aip/journal/jap/92/3?ver=pdfcov

Published by the AIP Publishing

\section{Articles you may be interested in}

Cross Sections for Electron Collisions with Methane

J. Phys. Chem. Ref. Data 44, 023101 (2015); 10.1063/1.4918630

Elastic and inelastic cross sections for low-energy electron collisions with pyrimidine

J. Chem. Phys. 136, 144310 (2012); 10.1063/1.3702629

Elastic Cross Sections for Electron Collisions with Molecules Relevant to Plasma Processing

J. Phys. Chem. Ref. Data 39, 033106 (2010); 10.1063/1.3475647

Absolute electron-impact total ionization cross sections of chlorofluoromethanes

J. Chem. Phys. 121, 11653 (2004); 10.1063/1.1808413

Evaluation of the computational methods for electron-impact total ionization cross sections: Fluoromethanes as benchmarks

J. Chem. Phys. 115, 4041 (2001); 10.1063/1.1388041

\section{AIP $\left.\right|_{\text {APL Photonics }}$}

APL Photonics is pleased to announce Benjamin Eggleton as its Editor-in-Chief

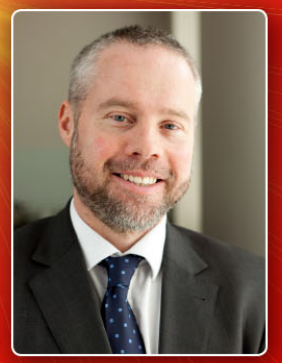




\title{
Electron collision cross sections for tetraethoxysilane
}

\author{
W. Lowell Morgan \\ Kinema Research and Software, P. O. Box 1147, 236 N. Washington Street, Monument, Colorado 80132 \\ Carl Winstead and Vincent McKoy \\ A. A. Noyes Laboratory of Chemical Physics, California Institute of Technology, Pasadena, \\ California 91125
}

(Received 25 February 2002; accepted for publication 10 May 2002)

\begin{abstract}
We describe the development of a consistent set of low-energy electron-collision cross sections for tetraethoxysilane, also known as tetraethylorthosilicate. Ab initio calculations are used to obtain the elastic and momentum-transfer cross sections. These are combined with measured values of the ionization cross section and with model vibrational and electronic excitation cross sections to form a preliminary cross section set, which is then adjusted to achieve consistency with measured swarm parameters. () 2002 American Institute of Physics. [DOI: 10.1063/1.1491024]
\end{abstract}

\section{INTRODUCTION}

Tetraethoxysilane, $\mathrm{Si}\left(\mathrm{OCH}_{2} \mathrm{CH}_{3}\right)_{4}$, also known as tetraethylorthosilicate and commonly called TEOS, is used in plasma deposition of $\mathrm{SiO}_{2}$ thin films in semiconductor device fabrication. Modeling and simulation of TEOS plasma chemistry requires knowledge of the relevant electron collision cross sections. Elastic or momentum-transfer cross sections are needed for the plasma electrical conductivity, ionization cross sections for the electron and ion densities, and dissociation cross sections for fragmentation of the parent gas into neutral radicals.

Here, we present electron collision cross sections for TEOS that are based on measurements, $a b$ initio calculations, and electron swarm analysis. We employ the approach that we have used in our previous work on $\mathrm{CHF}_{3},{ }^{1} \mathrm{C}_{2} \mathrm{~F}_{4}$, ,3 and $c-\mathrm{C}_{4} \mathrm{~F}_{8} \cdot{ }^{4,5}$ We obtain the elastic cross section for TEOS from an $a b$ initio calculation and then, making use of measured ionization cross sections, ${ }^{6,7}$ we employ swarm analysis techniques to derive two vibrational excitation cross sections and a total dissociation cross section.

The ab initio calculations are discussed in Sec. II. In Sec. III, we describe and present results from our swarm analyses, which yield the self-consistent cross section set that we seek.

\section{AB INITIO CALCULATIONS}

To obtain the equilibrium geometry of the TEOS ground electronic state, we optimized the internuclear distances and angles within the $S_{4}$ symmetry point group at the MP2/6$31 \mathrm{G}(d)$ level of approximation, using GAUSSIAN $94 .{ }^{8} \mathrm{Al}-$ though this commonly used approximation gives reasonably good geometries, it tends to overestimate bond lengths, which may in turn cause electron-scattering resonances to be shifted to lower energies. Elastic cross sections were calculated using the Schwinger multichannel (SMC) method ${ }^{9,10}$ as implemented for parallel computers. ${ }^{11,12}$ For the scattering calculations, we used the $6-311++\mathrm{G}(2 d, 2 p)$ basis set as defined in the program GAMESS ${ }^{13}$ with default splitting factors for the $d$ functions. Using this basis set, the total number of contracted Gaussian basis functions for TEOS was 525; after allowing for the 57 occupied molecular orbitals, 468 virtual orbitals were therefore available for description of the scattering electron. The SMC calculations were carried out at the static-exchange (SE) level of approximation, i.e., with polarization effects neglected. The principal limitations of the SE approximation occur at the lowest energies $(\leq 5 \mathrm{eV})$, where a spurious enhancement of $s$-wave scattering is seen, and in the presence of resonances, which tend to be placed too high in energy. Errors of the latter type should be partially offset by the use of MP2/6-31G $(d)$ bond lengths, although we still expect the calculated resonance energies to be too high.

In Fig. 1, we show the integral elastic cross section (ICS) and momentum-transfer cross section obtained from the calculation. Also shown are the contributions to the integral elastic cross section from different symmetry components, labeled according to the irreducible representations of $S_{4}$. The sharp dips near 28 and $40 \mathrm{eV}$ are clearly numerical in origin: they occur in multiple symmetries, and we have occasionally seen such features at the same energies in the cross sections of other large molecules; however, their precise origin is not known. Apart from these artifacts, the only prominent features of the ICS are a broad peak near $11.5 \mathrm{eV}$ and a rise in the cross section at the lowest energies $(~ \leq 2$ $\mathrm{eV}$ ), the latter due to the enhancement of $s$-wave scattering characteristic of the SE approximation.

Looking at the individual symmetry components instead of the summed ICS, it is possible to discern more structure. In particular, the shoulder that is barely visible near $4 \mathrm{eV}$ in the ICS turns out to arise from a peak of ${ }^{2} A$ symmetry at about $4.5 \mathrm{eV}$. There are also maxima in the ${ }^{2} E$ and ${ }^{2} B$ components at about 2.0 and $2.5 \mathrm{eV}$, respectively; however, from their shapes, these maxima appear to be nonresonant, representing instead the rapid turn on and subsequent slow falloff of $p$-wave scattering. Beneath the $11.5 \mathrm{eV}$ peak in the ICS are a ${ }^{2} B$ peak near $11 \mathrm{eV}$ and a ${ }^{2} A$ peak near $12 \mathrm{eV}$; however, there is also a very broad maximum in ${ }^{2} E$ extending from $\sim 6$ to $\sim 20 \mathrm{eV}$ that appears to comprise multiple overlapping resonances. A weak shoulder is also discernible near 19 


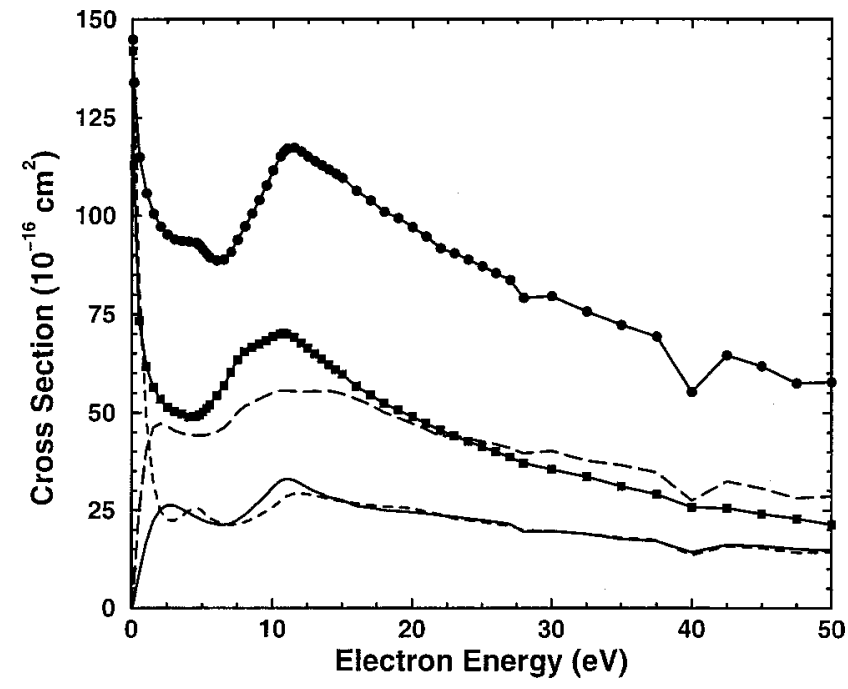

FIG. 1. Integral elastic cross section (solid line with circles) and momentum-transfer cross section (solid line with squares) for electron scattering by TEOS. Also shown are the symmetry components of the integral cross section: dotted line, ${ }^{2} A$; solid line, ${ }^{2} B$; dashed line, ${ }^{2} E$.

$\mathrm{eV}$ in ${ }^{2} A$ (and possibly ${ }^{2} B$ ). It may be noted that at higher energies, away from the influence of resonances, the contributions of the ${ }^{2} A$ and ${ }^{2} B$ components become nearly equal, while the contribution of the doubly degenerate ${ }^{2} E$ component is nearly equal to twice that of ${ }^{2} A$ or ${ }^{2} B$.

Although detailed exploration of the TEOS resonances is beyond the scope of the present article, we can gain some useful insights by examining the symmetries and energies of the virtual Hartree-Fock orbitals computed in a minimal basis set, because one generally finds a good correlation between the virtual valence orbitals and the low-energy shape resonances (though the orbital energies typically need to be shifted downward by several electronvolts). A calculation using the STO-6G minimal basis set and the same nuclear geometry as the scattering calculation places the lowest unoccupied molecular orbital (LUMO) of TEOS, which has $a$ symmetry, at $9.16 \mathrm{eV}$, with closely spaced $b$ and $e$ orbitals nearby, at 11.19 and $11.61 \mathrm{eV}$, respectively. Examination of their coefficients reveals that these orbitals all have considerable $\mathrm{Si}-\mathrm{O}$ antibonding character. The remaining 21 virtual orbitals occur in a band extending from 16.2 to $21.1 \mathrm{eV}$. For comparison, the STO-6G orbitals of ethanol range in energy from 15.0 to $20.9 \mathrm{eV}$, with the LUMO having considerable $\mathrm{O}-\mathrm{H}$ antibonding character.

In a recent study of $c-\mathrm{C}_{4} \mathrm{~F}_{8},{ }^{5}$ we found a shift of 4 to 5 $\mathrm{eV}$ between SE resonance energies and STO-6G orbital energies. If we apply the same shift here, the $a$ LUMO lines up closely with the $4.5 \mathrm{eV}^{2} A$ resonance, and the $\mathrm{Si}-\mathrm{O}$ antibonding $e$ orbital, shifted to near $7 \mathrm{eV}$, can account for the rise in ${ }^{2} E$ that begins above $6 \mathrm{eV}$, if we assume that the resonance it produces is broad and overlaps higher-lying ${ }^{2} E$ features. The band of high-energy virtual orbitals also lines up reasonably well with the overlapping ${ }^{2} A,{ }^{2} B$, and ${ }^{2} E$ maxima between about 11 and $16 \mathrm{eV}$. What appears to be missing is a ${ }^{2} B$ resonance near $6.5 \mathrm{eV}$. On the whole, however, agreement is reasonably good, and it seems clear that the $4.5 \mathrm{eV}$ resonance can be assigned to $\mathrm{a}^{2} \mathrm{~A}$ shape reso-

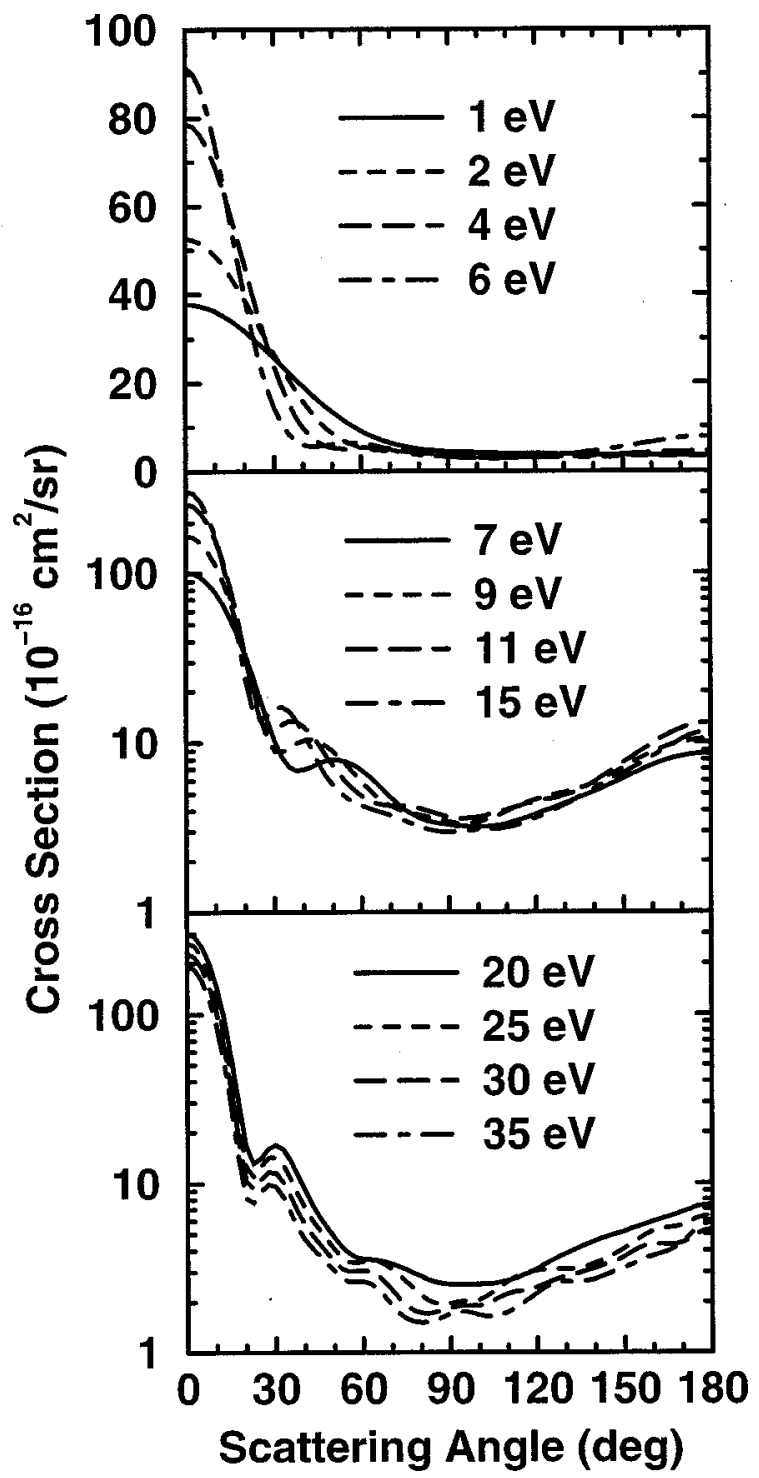

FIG. 2. Elastic differential cross section for scattering of electrons by TEOS at representative energies.

nance of $\mathrm{Si}-\mathrm{O}$ antibonding character, while the broad peak with maximum near $11.5 \mathrm{eV}$ is associated with resonances localized on the ethoxy moieties. These assignments give an indication of which modes may be involved in resonant vibrational excitation and, possibly, dissociative attachment. In each case, it should be borne in mind that the actual resonance positions are likely to be lower in energy than the SE positions.

In Fig. 2, we show the computed elastic differential cross section (DCS) at representative energies. One notable feature is how rapidly, as the energy increases, the DCS becomes forward peaked. Already at $6 \mathrm{eV}$, the DCS is dominated by a forward cone with the scattering angle $\vartheta \leq 30^{\circ}$. It is perhaps worth remarking that this forward peaking is not, as frequently seen in electron-molecule collisions, due to a strong molecular dipole moment-indeed, the $S_{4}$ geometry of TEOS implies a zero dipole moment - but reflects, rather, the geometric size of the TEOS molecule. As we might expect given the large nonresonant background and the multiplicity of overlapping resonances, no clear signals of the shape reso- 
nances seen in Fig. 1 are visible in the DCS; however, that the DCS at $11 \mathrm{eV}$ is more undulatory than the DCS at either 9 or $15 \mathrm{eV}$ probably reflects resonant effects.

To assist in construction of a cross section set, a vibrational spectrum for TEOS was computed using GAUSSIAN 94 at the restricted Hartree-Fock (RHF)/6-31G level of theory; as is usual, the resulting frequencies were scaled by 0.9 to account for the excessive stiffness of the RHF potential. We also computed energetics for dissociation processes, using MP2/6-311 $++\mathrm{G}(d, p)$ electronic energies obtained at the MP2/6-31G geometry together with RHF/6-31G frequencies; however, the number of processes considered was very limited owing to the size and low symmetry of the fragments involved.

\section{SWARM ANALYSIS AND RECOMMENDED CROSS- SECTION SET}

\section{A. Swarm analysis procedure}

We have available to us measurements ${ }^{14}$ of the drift velocity and ionization coefficient for electrons in TEOS. The electron drift velocity calculated from the solution $f_{0}(\varepsilon)$ of Boltzmann's equation is defined as

$$
V_{d}=\left\langle v_{z}\right\rangle=-\frac{1}{3}\left(\frac{2 e}{m}\right)^{1 / 2} \frac{E}{N} \int \frac{\mathrm{d} f_{0}(\varepsilon, E / N) / \mathrm{d} \varepsilon}{\sigma_{m}} \varepsilon \mathrm{d} \varepsilon,
$$

where $\varepsilon$ is the electron energy, $m$ is the electron mass, $E / N$ is the electric field divided by the gas number density $(\vec{E}=E \vec{z}), f_{0}$ is the electron energy distribution function, and $\sigma_{m}$ is the momentum-transfer cross section.

The ionization rate coefficient is defined as

$$
k_{i}=\left(\frac{2 e}{m}\right)^{1 / 2} \int \sigma_{i}(\varepsilon) f_{0}(\varepsilon) \varepsilon \mathrm{d} \varepsilon,
$$

where $\sigma_{i}$ is the ionization cross section. The ionization coefficient measured in a swarm experiment is

$$
\alpha=\frac{k_{i} N}{V_{d}},
$$

which is the increase in electron density per centimeter due to ionization as a swarm of electrons drifts against an electric field. This is usually displayed as $\alpha / N$ with units of $\mathrm{cm}^{2}$.

We used a cross section set consisting of the ab initio momentum-transfer cross section, the measured ionization cross section, and model cross sections for vibrational excitation and for dissociation. We then adjusted the magnitudes of the model cross sections in order for the calculated swarm coefficients to agree with the measurements. Boltzmann's equation for the transport of electrons in TEOS was solved using the two-term spherical harmonic expansion ${ }^{15}$ for low values of $E / N$ and Monte Carlo simulation for large $E / N$, and the model cross sections were adjusted by means of the downhill simplex optimization algorithm. ${ }^{16-19}$ Although, as noted in Sec. II, the SE momentum-transfer cross section is expected to be overly large at low energies, the transport parameters are fairly insensitive to the low-energy region; consequently, it was not necessary to adjust the calculated momentum transfer cross section.

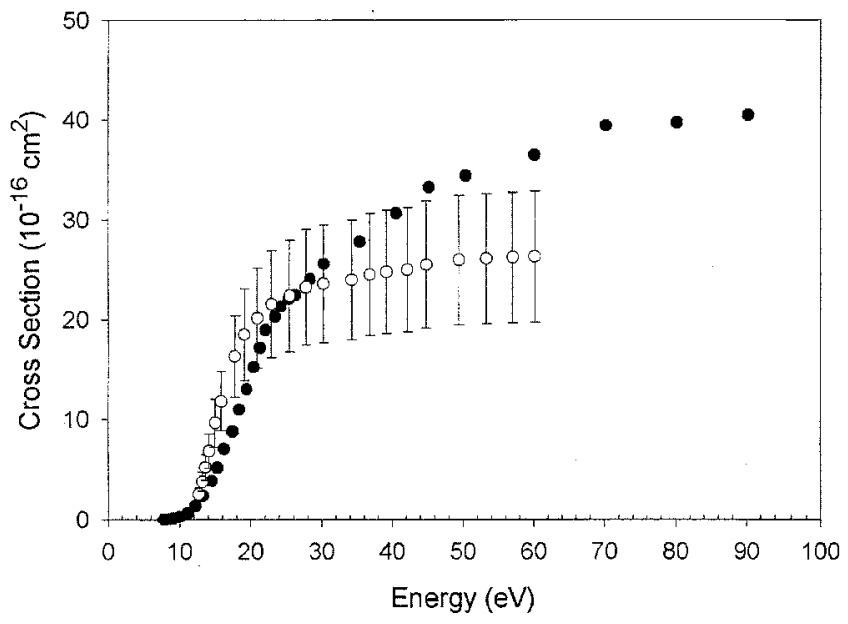

FIG. 3. Electron-impact total ionization cross sections for TEOS. The open circles with error bars are the measurements of Holtgrave et al., ${ }^{6}$ and the solid circles are those of Basner et al. ${ }^{7}$

There have been two measurements of the total and partial ionization cross sections for TEOS. The total cross sections are shown in Fig. 3. We have used the more recent measurements of Becker et al. ${ }^{7}$ in our calculations.

As we have discussed in detail elsewhere, ${ }^{2}$ the two-term spherical harmonic expansion solution to Boltzmann's equation begins to fail for values of $E / N$ in excess of, say, 500$1000 \mathrm{Td}$. We therefore have employed Monte Carlo simulation of electron transport for high values of $E / N$. We use the total elastic cross section and the differential elastic cross sections for TEOS in these calculations.

The energy dependence of the model vibrational excitation cross sections is based on the Born approximation. ${ }^{20}$ If $\Delta \varepsilon$ is the vibrational excitation energy and $\varepsilon$ is the electronimpact energy, the model vibrational excitation cross section is

$$
\sigma_{v}^{B}=\frac{\sigma_{0}}{\Delta \varepsilon x} \ln \left(\frac{x^{1 / 2}+(x-1)^{1 / 2}}{\left|x^{1 / 2}-(x-1)^{1 / 2}\right|}\right),
$$

where $x=\varepsilon / \Delta \varepsilon$ and the factor $\sigma_{0}$ is to be determined by the swarm analysis. Were we concerned with detailed lowenergy cross sections, we would need to account for nondipole-allowed vibrational excitations, whose cross sections fall off more quickly from threshold than do the Born profiles we have assumed.

The vibrational energy spectrum of TEOS is shown in Fig. 4. We have chosen to use two model cross sections having excitation energies of 0.16 and $0.37 \mathrm{eV}$ to represent the numerous levels shown in Fig. 4. Figure 4 also shows the fractional populations of the vibrational levels at a temperature of $300 \mathrm{~K}$. Clearly, there is substantial excitation of the continuum of low-lying vibrational levels. Again, were we concerned with detailed low-energy cross sections, we would need to account for electron superelastic collisions, whereby electrons gain energy in collisions with vibrationally excited states, in our solution of Boltzmann's equation.

For the model dissociation cross section, we assumed the form 


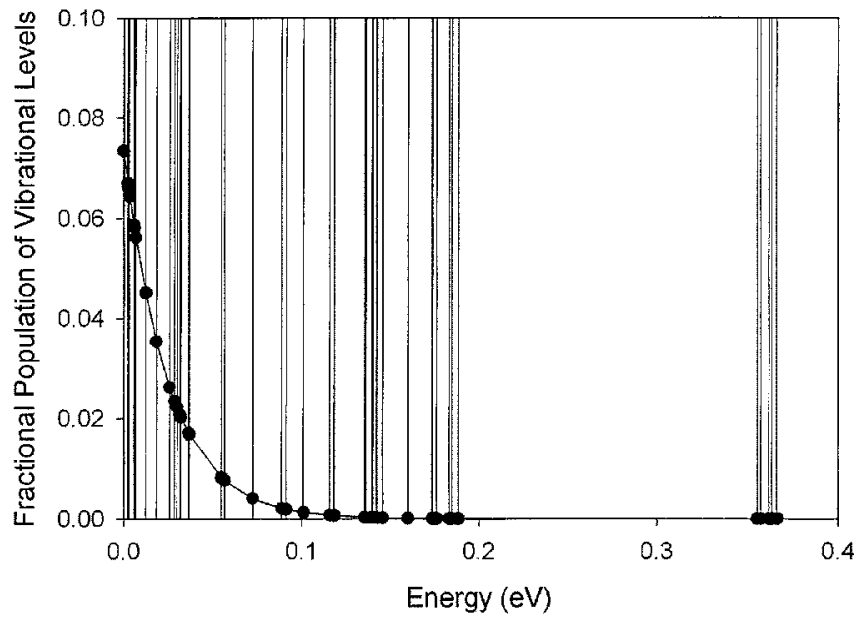

FIG. 4. The 94 lowest vibrational energies of TEOS. The solid circles show the fractional populations of the vibrational states at a temperature of $300 \mathrm{~K}$.

$$
\sigma_{d}(\varepsilon)=\sigma_{d}^{0}\left(\frac{1-1 / x}{x}\right),
$$

where $x=\varepsilon / \Delta \varepsilon$, with $\Delta \varepsilon$ now indicating the dissociation threshold. The dissociation energetics of TEOS are shown in Table I. Although the adiabatic thresholds shown in Table I range as low as $3.6 \mathrm{eV}$, they are not necessarily indicative of the vertical thresholds that would be observed in an impulsive process such as electron-impact excitation. In fact, the lowest vertical threshold obtained from a single-excitation configuration-interaction calculation within the $6-31+\mathrm{G}(d)$ basis set at the MP2/6-31G $(d)$ geometry proved to be $9.0 \mathrm{eV}$, for a state of ${ }^{3} E$ symmetry. In constructing the TEOS cross section set, we adjusted $\Delta \varepsilon$ for dissociation and found that $7.0 \mathrm{eV}$ gave the best fit to the swarm data.

\section{B. Results of swarm analysis}

Our final set of cross sections is shown in Fig. 5, while Fig. 6 shows the measured ${ }^{14}$ electron drift velocity and ionization coefficient over a range of $E / N$ from 12 to $6000 \mathrm{Td}$ along with the results of our calculations of the transport coefficients. The results from the numerical solution to Boltzmann's equation cover the range of $E / N$ from 20 to 1000 $\mathrm{Td}$, and the Monte Carlo results range from 700 to $6000 \mathrm{Td}$. The error bars on the measured drift velocity range from $\pm 2 \%$ at $10 \mathrm{Td}$ to about $\pm 5 \%$ at $1000 \mathrm{Td}$ and are greatest at $6000 \mathrm{Td}$, with values of $\pm 7 \%$ there. The error bars on the measured ionization coefficient are about $\pm 32 \%$ at $500 \mathrm{Td}$ and narrow to $\pm 10 \%$ at $6000 \mathrm{Td}$.

Considering the simplicity of our model cross section set, our calculated transport coefficients agree reasonably well with the measurements. As we noted above, the numeri-

TABLE I. Dissociation energetics of TEOS

\begin{tabular}{lc}
\hline \hline \multicolumn{1}{c}{ Products } & Energy $(\mathrm{eV})$ \\
\hline $\mathrm{SiOCH}_{2}\left(\mathrm{OC}_{2} \mathrm{H}_{5}\right)_{3}+\mathrm{CH}_{3}$ & 3.6 \\
$\mathrm{SiO}\left(\mathrm{OC}_{2} \mathrm{H}_{5}\right)_{3}+\mathrm{C}_{2} \mathrm{H}_{5}$ & 4.0 \\
$\mathrm{Si}\left(\mathrm{OC}_{2} \mathrm{H}_{5}\right)_{3}+\mathrm{C}_{2} \mathrm{H}_{5} \mathrm{O}$ & 5.2 \\
\hline \hline
\end{tabular}

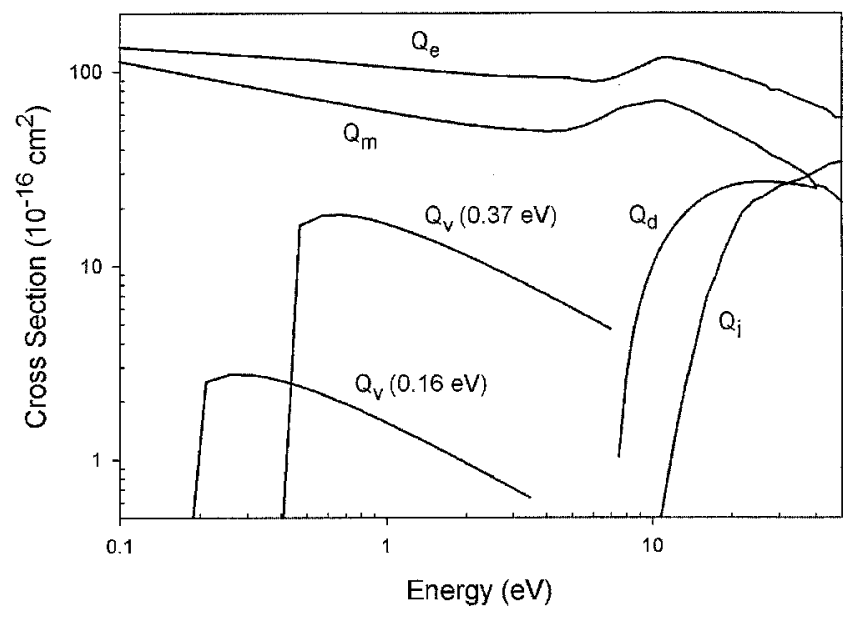

FIG. 5. Cross section set for TEOS. $Q_{e}$ and $Q_{m}$ are the integrated $a b$ initio cross sections described in Sec. II.

cal solution of Boltzmann's equation begins to fail at values of $E / N$ greater than about $500 \mathrm{Td}$. This occurs as the drift velocity becomes non-negligible compared to the thermal velocity, violating the assumptions underlying the two-term spherical harmonic expansion of Boltzmann's equation.

\section{SUMMARY}

We have presented the results of $a b$ initio calculations of the elastic and momentum-transfer cross sections for the collisions of electrons with the TEOS molecule. Making use of the calculated cross sections as well as measured ionization cross sections and measured electron-transport coefficients, we have constructed a self-consistent model cross section set that includes vibrational excitation cross sections and a total electronic excitation or dissociation cross section. This cross section set should provide an adequate starting point for modeling the chemistry of plasmas containing TEOS as a constituent.

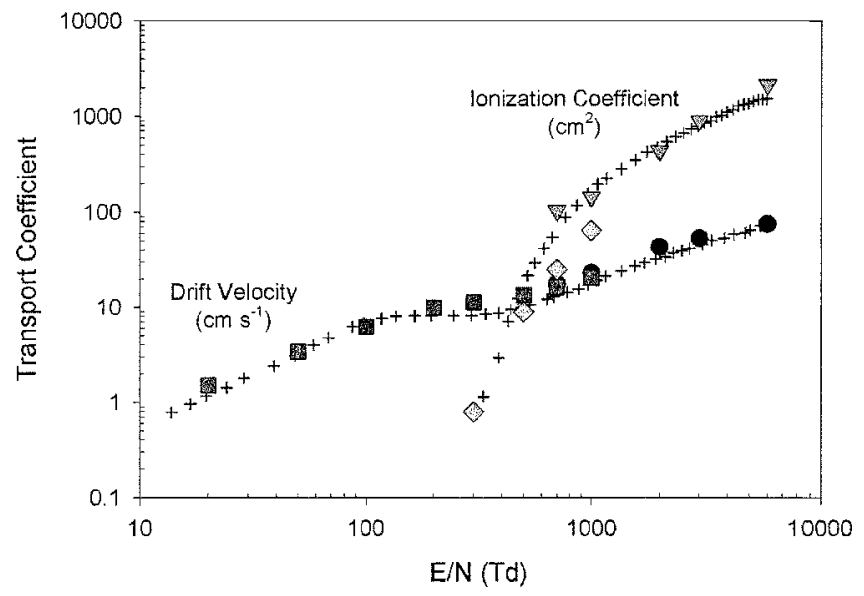

FIG. 6. Calculated drift velocities $V_{d}$ and ionization coefficients $\alpha / N$ for electrons in TEOS. The filled squares are $V_{d}$ calculated using a numerical solution of Boltzmann's equation. The filled circles are results of Monte Carlo simulations. The diamonds are $\alpha / N$ from solving Boltzmann's equation and the triangles are the Monte Carlo results. Experimental values ${ }^{14}$ are shown by the + signs. 


\section{ACKNOWLEDGMENTS}

This work was supported by SEMATECH, Inc. Work at the California Institute of Technology was also supported by the Department of Energy, Office of Basic Energy Sciences, and by an equipment grant from Intel Corp. Cross section calculations made use of the facilities of the Caltech Center for Advanced Computational Research, which is supported in part by the National Science Foundation.

${ }^{1}$ W. L. Morgan, C. Winstead, and V. McKoy, J. Appl. Phys. 90, 2009 (2001).

${ }^{2}$ K. Yoshida, S. Goto, H. Tagashira, C. Winstead, V. McKoy, and W. L. Morgan, J. Appl. Phys. 91, 2637 (2002).

${ }^{3}$ C. Winstead and V. McKoy, J. Chem. Phys. 116, 1380 (2002).

${ }^{4}$ G. I. Font, W. L. Morgan, and G. Mennenga, J. Appl. Phys. 91, 3530 (2002).

${ }^{5}$ C. Winstead and V. McKoy, J. Chem. Phys. 114, 7407 (2001).

${ }^{6}$ J. Holtgrave, K. Riehl, D. Abner, and P. D. Haaland, Chem. Phys. Lett. 215, 548 (1993).

${ }^{7}$ R. Basner, M. Schmidt, K. Becker, and H. Deutsch, Adv. At., Mol., Opt. Phys. 43, 147 (2000).

${ }^{8}$ M. J. Frisch, G. W. Trucks, H. B. Schlegel, P. M. W. Gill, B. G. Johnson, M. A. Robb, J. R. Cheeseman, T. Keith, G. A. Petersson, J. A. Montgomery, K. Raghavachari, M. A. Al-Laham, V. G. Zakrzewski, J. V. Ortiz, J. B.
Foresman, J. Cioslowski, B. B. Stefanov, A. Nanayakkara, M. Challacombe, C. Y. Peng, P. Y. Ayala, W. Chen, M. W. Wong, J. L. Andres, E. S. Replogle, R. Gomperts, R. L. Martin, D. J. Fox, J. S. Binkley, D. J. Defrees, J. Baker, J. P. Stewart, M. Head-Gordon, C. Gonzalez, and J. A. Pople, Gaussian 94, Revision C.4 (Gaussian, Inc., Pittsburgh, PA, 1995).

${ }^{9}$ K. Takatsuka and V. McKoy, Phys. Rev. A 24, 2473 (1981).

${ }^{10}$ K. Takatsuka and V. McKoy, Phys. Rev. A 30, 1734 (1984).

${ }^{11}$ C. Winstead and V. McKoy, Adv. At., Mol., Opt. Phys. 36, 183 (1996).

${ }^{12}$ C. Winstead and V. McKoy, Comput. Phys. Commun. 128, 386 (2000).

${ }^{13}$ M. W. Schmidt, K. K. Baldridge, J. A. Boatz, S. T. Elbert, M. S. Gordon, J. H. Jensen, S. Koseki, N. Matsunaga, K. A. Nguyen, S. J. Su, T. L. Windus, M. Dupuis, and J. A. Montgomery, J. Comput. Chem. 14, 1347 (1993).

${ }^{14}$ K. Yoshida, T. Ohshima, H. Ohuchi, and H. Tagashira, J. Phys. D 28, 2478 (1995).

${ }^{15}$ W. L. Morgan and B. M. Penetrante, Comput. Phys. Commun. 58, 127 (1990).

${ }^{16}$ W. H. Press, S. A. Teukolsky, W. T. Vetterling, and B. P. Flannery, Numerical Recipes in Fortran 77, 2nd ed. (Cambridge University Press, New York, 1992).

${ }^{17}$ N. Gershenfeld, The Nature of Mathematical Modeling (Cambridge University Press, New York, 1999).

${ }^{18}$ W. L. Morgan, Phys. Rev. A 44, 1677 (1991).

${ }^{19}$ W. L. Morgan, J. Phys. D 26, 209 (1993).

${ }^{20}$ N. F. Lane, Rev. Mod. Phys. 52, 29 (1980). 